\title{
Association of incident obstructive sleep apnoea with outcomes in a large cohort of US veterans
}

\author{
Miklos Z Molnar, ${ }^{1}$ Istvan Mucsi, ${ }^{2,3}$ Marta Novak, ${ }^{3,4}$ Zoltan Szabo, ${ }^{5,6}$ \\ Amado X Freire, ${ }^{7}$ Kim M Huch, ${ }^{8}$ Onyebuchi A Arah, ${ }^{9}$ Jennie Z Ma, ${ }^{10}$ Jun L Lu, ${ }^{1}$ \\ John J Sim, ${ }^{11}$ Elani Streja, ${ }^{12}$ Kamyar Kalantar-Zadeh, ${ }^{12}$ Csaba P Kovesdy ${ }^{1,8}$
}

\begin{abstract}
- Additional material is published online only. To view this file please visit the journal online (http://dx.doi.org/10. 1136/thoraxjn-2015-206970).

For numbered affiliations see end of article.
\end{abstract}

\section{Correspondence to}

Dr Miklos Z Molnar, Division of Nephrology, Department of Medicine, University of Tennessee Health Science Center, 956 Court Ave, Suite B216B, Memphis, TN 38163, USA; mzmolnar@uthsc.edu

Received 24 February 2015 Revised 23 April 2015 Accepted 9 May 2015 Published Online First 2 June 2015

\section{SLinked}

- http://dx.doi.org/10.1136/ thoraxjnl-2014-206655

- http://dx.doi.org/10.1136/ thoraxjnl-2015-206871

- http://dx.doi.org/10.1136/

thoraxjnl-2015-207247

\section{CrossMark}

To cite: Molnar MZ,

Mucsi I, Novak M, et al.

Thorax 2015;70:888-895.

\section{ABSTRACT}

Rationale There is a paucity of large cohort studies examining the association of obstructive sleep apnoea (OSA) with clinical outcomes including all-cause mortality, coronary heart disease (CHD), strokes and chronic kidney disease (CKD).

Objectives We hypothesised that a diagnosis of incident OSA is associated with higher risks of these adverse clinical outcomes.

Methods, measurements In a nationally representative cohort of over 3 million ( $n=3079$ 514) US veterans $(93 \%$ male) with baseline estimated glomerular filtration rate (eGFR) $\geq 60 \mathrm{~mL} / \mathrm{min} / 1.73 \mathrm{~m}^{2}$, we examined the association between the diagnosis of incident OSA, treated and untreated with CPAP, and: (1) all-cause mortality, (2) incident CHD, (3) incident strokes, (4) incident CKD defined as eGFR $<60 \mathrm{~mL} / \mathrm{min} / 1.73 \mathrm{~m}^{2}$, and (5) slopes of eGFR.

Main results Compared with OSA-negative patients, untreated and treated OSA was associated with $86 \%$ higher mortality risk, (adjusted HR and $95 \% \mathrm{Cl} 1.86$ (1.81 to 1.91$)$ and $35 \%$ (1.35 (1.21 to 1.51$)$ ), respectively. Similarly, untreated and treated OSA was associated with 3.5 times (3.54 (3.40 to 3.69)) and 3 times (3.06 (2.62 to 3.56)) higher risk of incident CHD; 3.5 times higher risk of incident strokes (3.48 (3.28 to 3.64) and 3.50 (2.92 to 4.19)) for untreated and treated OSA, respectively. The risk of incident CKD was also significantly higher in untreated (2.27 (2.19 to 2.36)) and treated (2.79 (2.48 to 3.13)) patients with OSA. The median (IQR) of the eGFR slope was $-0.41(-2.01$ to 0.99$),-0.61(-2.69$ to 0.93$)$ and $-0.87(-3.00$ to $0.70) \mathrm{mL} / \mathrm{min} / 1.73 \mathrm{~m}^{2}$ in OSA-negative patients, untreated OSA-positive patients and treated OSA-positive patients, respectively.

Conclusions In this large and contemporary cohort of more than 3 million US veterans, a diagnosis of incident OSA was associated with higher mortality, incident CHD, stroke and CKD and with faster kidney function decline.

\section{INTRODUCTION}

Obstructive sleep apnoea (OSA) is one of the most clinically important forms of sleep-related breathing disorders. The prevalence of moderate and severe obstructive OSA, defined as an apnoea hypopnoea index (AHI) $>15$ and the presence of daytime symptoms of OSA is $10 \%$ in the general population. ${ }^{1}$

Previous studies have shown that OSA is associated with a higher risk of mortality, ${ }^{2-4}$ but some studies did not confirm this association in elderly

\section{Key messages}

What is the key question?

- We assessed the association between a diagnosis of incident obstructive sleep apnoea (OSA) and adverse clinical outcomes such as incident chronic kidney disease (CKD) and mortality.

\section{What is the bottom line?}

- In this large and contemporary cohort of more than 3 million US veterans, a diagnosis of incident OSA was associated with higher mortality, incident coronary heart disease, stroke and CKD, and faster kidney function decline.

\section{Why read on?}

- This is the first study to show an association of a diagnosis of incident OSA with CKD and faster kidney function decline.

patients with prevalent OSA. ${ }^{5-7}$ In addition to increased mortality risk, OSA has also shown positive associations with cardiovascular and cerebrovascular events. ${ }^{3}{ }^{8-10}$ However, most of these studies were limited by small sample size and low event rates. ${ }^{9} 11$

In patients with chronic kidney disease (CKD), OSA is associated with accelerated atherosclerosis, hypertension and vascular damage. ${ }^{12}$ The complex pathophysiology that links OSA to cardiovascular risk may also have a detrimental effect on kidney function. Patients with OSA experience glomerular hyperfiltration, which is alleviated by short-term CPAP treatment, ${ }^{13}$ but our previous studies in kidney transplant recipients found no association between OSA and deterioration of kidney function. ${ }^{14} 15$ Only one recently published study assessed the association of OSA with kidney function decline and progression to CKD in non-transplant patients. ${ }^{16}$

We examined the associations of incident treated and untreated OSA diagnosis with all-cause mortality, the incidences of coronary heart disease (CHD), ischaemic stroke and CKD, and the rate of kidney function decline in a large, nationally representative contemporary cohort of US veterans. Based on previous findings, we hypothesised that a diagnosis of incident OSA is associated with higher risks of these adverse clinical outcomes. 


\section{METHODS}

\section{Study setting and cohort definition}

Data were obtained from the Racial and Cardiovascular Risk Anomalies in CKD study, which examines risk factors in patients with incident CKD in US veterans, and which was previously described in detail. ${ }^{17}{ }^{18}$ Diagnoses of incident OSA, CPAP and polysomnography (overnight sleep study) were identified from the VA Inpatient and Outpatient Medical SAS data sets using ICD-9-CM diagnostic and procedure codes and Current Procedural Terminology (CPT) codes (see online supplementary table S1). ${ }^{19}$ The algorithm for cohort definition is shown in figure 1. Patients were included in the study if they had a baseline estimated glomerular filtration rate (eGFR) $\geq 60 \mathrm{~mL} / \mathrm{min} /$ $1.73 \mathrm{~m}^{2}$ and did not have a diagnosis of OSA at the first encounter in the inclusion period (1 October 2004-30 September 2006), while other comorbidities were listed in that encounter. The final cohort included 3079514 patients.

\section{Exposure and covariates}

Incident OSA was defined as a new ICD9-CM code for OSA during the inclusion period, without such a diagnosis at the first encounter of the inclusion period. Among the 3079514 patients, 21764 had incident diagnosis of OSA but without CPAP treatment (OSA+/CPAP-) and 1478 had incident diagnosis of OSA treated with CPAP (OSA+/CPAP+) (figure 1).

Sociodemographic characteristics, comorbid conditions and laboratory characteristics were obtained, as previously described. $^{20-23}$ Information about age, gender and race were obtained through the VA Corporate Data Warehouse and from Medicare through the VA-Medicare data merge project. ${ }^{24}$ Information about comorbidities was collected from the VA Inpatient and Outpatient Medical SAS data sets using ICD-9-CM diagnostic and procedure codes and CPT codes (see online supplementary table S2). ${ }^{19}$ Prevalent comorbidities were defined as those diagnosed during 1 October 2004-30 September 2006.

\section{Outcomes}

We defined five different outcomes: (1) all-cause mortality, (2) incident CHD, (3) incident ischaemic stroke, (4) incidence of CKD and (5) slopes of eGFR.

Data on all-cause mortality was obtained from the VA Vital Status Files, which contain dates of death or last medical/

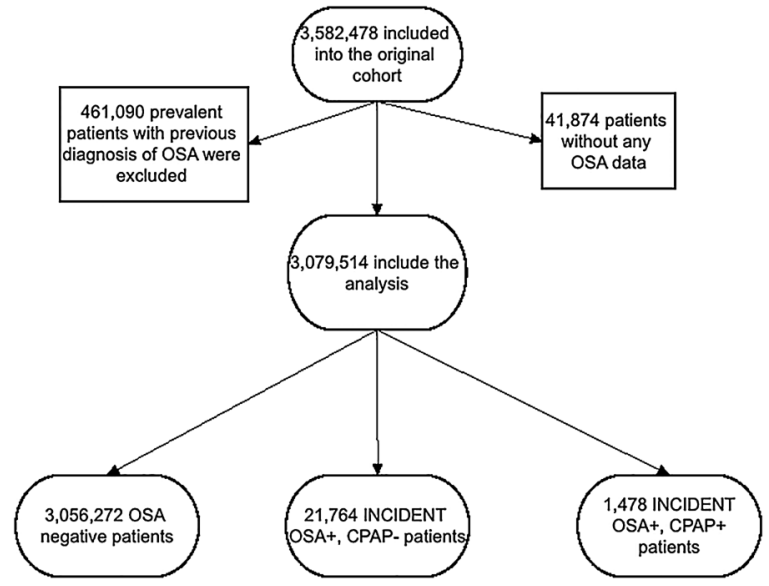

Figure 1 Flow chart of patients' selection. OSA, obstructive sleep apnoea. administrative encounter from all sources in the VA system with sensitivity and specificity of $98.3 \%$ and $99.8 \%$, respectively, as compared with the National Death Index. Incident CHD was defined as the composite outcome of a first occurrence of an ICD-9-CM or CPT code for acute myocardial infarction, coronary artery bypass grafting or percutaneous angioplasty after 1 October 2006 in patients without such diagnoses prior to this date. Incident stroke was defined as the first occurrence of ICD-9-CM codes for ischaemia after 1 October 2006 in patients without such diagnoses prior to this date (see online supplementary tables S3 and S4). Incident CKD was defined as two consecutive eGFR levels $<60 \mathrm{~mL} / \mathrm{min} / 1.73 \mathrm{~m}^{2}$ separated by $\geq 90$ days, and a $>25 \%$ decrease from baseline eGFR. eGFR was calculated from serum creatine measurements using the Chronic Kidney Disease Epidemiology Collaboration Equation. Slopes of eGFR were calculated in each patient from all available eGFR values derived from outpatient serum creatine measurements and using least squares regression. The median (IQR) number of serum creatine measurements used to calculate eGFR slopes was 10 (5-17). Rapid deterioration was defined as slopes of $<-5 \mathrm{~mL} / \mathrm{min} / 1.73 \mathrm{~m}^{2} /$ year (ie, loss of eGFR of $>5 \mathrm{~mL} / \mathrm{min} /$ $1.73 \mathrm{~m}^{2} /$ year).

\section{Statistical analysis}

Data were summarised using proportions, means \pm SD or median (IQR) as appropriate. Continuous variables were compared using the Student's $t$ test and Mann-Whitney $U$ test according to data type. Predictors of incident diagnosis of OSA were assessed using logistic regression analyses. The associations between diagnoses of incident OSA-with and without CPAP treatment-with outcomes were assessed using the Kaplan-Meier method and Cox proportional hazard models (for time-to-event analyses) and logistic regressions (for slopes).

The start of the follow-up period was the date of the first eGFR $\geq 60 \mathrm{~mL} / \mathrm{min} / 1.73 \mathrm{~m}^{2}$ during 1 October 2004-30 September 2006. Patients diagnosed with OSA at a subsequent date were considered as part of the non-OSA group for the time period between cohort entry and diagnosis of OSA. Patients were followed until different end points or were censored at the date of last healthcare or administrative visit, or on 26 July 2013.

In postevent (CHD or stroke) mortality analyses, the start of the follow-up period was the date of the event. Incident CHD and stroke events were identified after 1 October 2006 in patients without such diagnoses prior to this date; therefore, to avoid immortal time bias, the start of the follow-up period for these end points was 1 October 2006.

All associations were examined in unadjusted and adjusted models. Models were adjusted for the following confounders based on a priori considerations: model 1: age, gender, race/ethnicity; model 2: model 1 variables and baseline eGFR; model 3: model 2 variables and comorbidities at baseline (diabetes, hypertension, cardiovascular disease, congestive heart failure (CHF), cerebrovascular disease, peripheral vascular disease, lung disease, dementia, rheumatic disease, malignancy, HIV/AIDS and depression) and measures of quality of care (number of administered cholesterol measurements and influenza vaccinations); and model 4: model 3 variables and income, marital status and body mass index (BMI). The final fully adjusted model 4 analyses were repeated in different a priori selected subgroups.

Several sensitivity analyses were also performed. We repeated all analyses in a cohort of patients where the OSA diagnosis was 
based on the above-mentioned ICD-9 codes and confirmed by polysomnography. Because death and other outcomes are competing events, a competing risk regression model was used in a propensity-matched cohort of patients $(n=42340)$ with and without a diagnosis of incident OSA. Our events of interest were incident $\mathrm{CHD}$, ischaemic stroke and CKD and the competing event was death (see online supplementary table S5). In our study we used the Fine and Gray method, ${ }^{25}$ which extends the Cox proportional hazards model to competing-risks data by considering the subdistribution hazard. The competing risk models' goal was to estimate the cumulative incidence function, which is defined by the probability of events (see online supplementary figure S1).

Statistical analyses were performed using Stata MP V.12.0 (Stata Corporation, College Station, Texas, USA).

\section{RESULTS}

\section{Baseline characteristics}

The mean \pm SD age of the cohort at baseline was $60.5 \pm 14.4$ years, $93 \%$ were male, $79 \%$ and $17 \%$ of patients were white and black, respectively, 22\% were patients with diabetes and the mean baseline eGFR was $83.6 \pm 15.4 \mathrm{~mL} / \mathrm{min} / 1.73 \mathrm{~m}^{2}$. Baseline characteristics of patients categorised by OSA status are shown in table 1 . Patients with a diagnosis of incident OSA were slightly younger, more likely to be divorced and to have lower income, and had higher BMI, higher prevalence of hypertension, diabetes mellitus, CVD, CHF, cerebrovascular disease, depression and higher eGFR.

\section{Predictors of the diagnosis of incident OSA}

In our adjusted logistic regression model, younger age, male gender, caucasian, unmarried status, higher BMI and most of

Table 1 Baseline characteristics of study population

\begin{tabular}{|c|c|c|c|}
\hline & OSA-negative (n=3 056 272) & $\begin{array}{l}\text { OSA-positive without } \\
\text { treatment }(n=21764)\end{array}$ & $\begin{array}{l}\text { OSA-positive with } \\
\text { treatment }(n=1478)\end{array}$ \\
\hline Age (years) & $61 \pm 14$ & $59 \pm 11$ & $57 \pm 10$ \\
\hline Gender (male) & $2840692(93)$ & 20932 (96) & $1421(96)$ \\
\hline \multicolumn{4}{|l|}{ Outcomes } \\
\hline Death & $671645(22)$ & $5996(28)$ & $323(22)$ \\
\hline Incident CHD event* & $68268(2)$ & $2537(12)$ & $167(12)$ \\
\hline Incident stroke eventt & $50333(2)$ & $1601(8)$ & $122(9)$ \\
\hline New CKD & $290037(10)$ & $5486(25)$ & $434(29)$ \\
\hline \multicolumn{4}{|l|}{ Race } \\
\hline White & 2153744 (79) & $17020(80)$ & $1082(74)$ \\
\hline African-American & $465810(17)$ & 3402 (16) & $333(23)$ \\
\hline Hispanic & $63138(2)$ & $413(2)$ & $16(1)$ \\
\hline Other race & $57445(2)$ & $410(2)$ & $25(2)$ \\
\hline \multicolumn{4}{|l|}{ Marital status } \\
\hline Married & $1606708(55)$ & $10341(50)$ & $735(52)$ \\
\hline Single & $335237(11)$ & $2112(10)$ & $166(12)$ \\
\hline Divorced & $749305(26)$ & $6858(33)$ & $435(31)$ \\
\hline Widow & $229756(8)$ & $1538(7)$ & $77(5)$ \\
\hline \multicolumn{4}{|l|}{ Other sociodemographic } \\
\hline Income (USD) & 22849 (11 522-36 929) & $20327(11563-31$ 104) & 19881 (11 805-30 893) \\
\hline Service connection & $1154717(38)$ & $11208(51)$ & $778(53)$ \\
\hline Baseline eGFR $\left(\mathrm{mL} / \mathrm{min} . / 1.73 \mathrm{~m}^{2}\right)$ & $84 \pm 15$ & $85 \pm 15$ & $87 \pm 16$ \\
\hline BMI $\left(\mathrm{kg} / \mathrm{m}^{2}\right)$ & $28.3 \pm 4.9$ & $32.0 \pm 5.8$ & $33.1 \pm 5.4$ \\
\hline \multicolumn{4}{|l|}{ Comorbidities } \\
\hline Hypertension & $1763151(58)$ & $15881(73)$ & $1124(76)$ \\
\hline Diabetes mellitus & $674318(22)$ & $8675(40)$ & $644(44)$ \\
\hline Cardiovascular disease $\ddagger$ & $333638(11)$ & 4105 (19) & $308(21)$ \\
\hline Congestive heart failure & 121312 (4) & $2691(12)$ & $202(14)$ \\
\hline Cerebrovascular disease & $186119(6)$ & $1910(9)$ & $146(10)$ \\
\hline Peripheral arterial disease & $164507(5)$ & $1959(9)$ & $140(9)$ \\
\hline Chronic lung disease & 524645 (17) & $6592(30)$ & $450(30)$ \\
\hline Dementia & $27440(0.9)$ & $190(0.9)$ & $12(0.8)$ \\
\hline Rheumatological disease & $42154(1.4)$ & $370(1.7)$ & $30(2.0)$ \\
\hline Peptic ulcer disease & $56591(1.8)$ & $502(2.3)$ & $34(2.3)$ \\
\hline Liver disease & $37795(1.2)$ & $411(1.9)$ & $22(1.5)$ \\
\hline All malignancies & $327064(11)$ & $2296(11)$ & $138(9)$ \\
\hline AIDS/HIV & $19958(0.6)$ & $104(0.5)$ & $5(0.3)$ \\
\hline Depression & $249958(8)$ & 3325 (15) & $294(20)$ \\
\hline
\end{tabular}

Dichotomous/dummy variables are presented as number of patients and percentage; continuous variables are presented as mean \pm SD or median (IQR).

${ }^{*}$ In cardiovascular disease-free (see below) patients at baseline.

tIn stroke disease-free patients at baseline.

¥Cardiovascular disease was defined as acute myocardial infraction, angina, coronary artery disease, previous coronary artery bypass grafting or percutaneous coronary intervention.

$\mathrm{BMI}$, body mass index; CHD, coronary heart disease; CKD, chronic kidney disease; eGFR, estimated glomerular filtration rate; OSA, obstructive sleep apnoea. 
the comorbidities (such as diabetes, hypertension, CVD, CHF) were associated with the presence of a diagnosis of incident OSA (table 2).

\section{Mortality}

The median follow-up time was 7.74 years (IQR: 5.998.37 years). There were 671645 deaths (22\%, mortality rate $32.6(32.5-32.7) / 1000$ patient-years) in the OSA-negative group, 323 deaths $(22 \%, 62.3$ (60.7-63.9)/1000 patient-years) in the treated OSA group and 5996 deaths $(28 \%, 39.9$ (35.844.5)/1000 patient-years) in the untreated OSA group. Figures 2 and 3 show the associations between the diagnosis of incident OSA and mortality in unadjusted and adjusted models. Untreated OSA was associated with higher mortality in the unadjusted (HR: $1.86,95 \%$ CI 1.81 to 1.91 ) and fully adjusted models (HR: 1.86, 95\% CI 1.81 to 1.91) (figure 3A). Treated OSA was also associated with higher mortality in the unadjusted (HR: $1.20,95 \%$ CI 1.07 to 1.33 ) and fully adjusted models (HR: $1.35,95 \%$ CI 1.21 to 1.51 ) (figure 3B). Similar results were present in all subgroups in untreated OSA-positive patients and most of the treated OSA-positive subgroup (see online supplementary figure $\mathrm{S} 1$ ).

Figure 4 shows the association between a diagnosis of incident OSA and postevent mortality. Untreated OSA was associated with significantly higher post-CHD event mortality only in the fully adjusted model (HR: $1.23,95 \%$ CI 1.14 to 1.33 ). However, the post-CHD event mortality was similar between OSA-negative and treated OSA-positive patients (figure 4). Compared with OSA-negative patients, patients with untreated OSA exhibited a trend towards higher poststroke mortality in the unadjusted model (HR: 1.06, 95\% CI 0.97 to 1.16), and a significantly higher poststroke mortality in the fully adjusted model (HR: 1.21, 95\% CI 1.10 to 1.33) (figure 4). However, the poststroke mortality risk was similar between OSA-negative and treated OSA-positive patients (figure 4).

\section{Incident CHD}

There were 68268 incident CHD events (2.3\%, event rate 4.17 (4.14-4.20)/1000 patient-years) in the OSA-negative group, 167 incident CHD events $(12.1 \%, 20.1$ (17.2-23.4)/1000 patientyears) in the treated OSA group and 2537 incident CHD events $(12.4 \%, 21.5$ (20.7-22.3)/1000 patient-years) in the untreated OSA group. Figures 2 and 3 show the associations between a diagnosis of incident OSA and incident CHD events in unadjusted and adjusted models. Untreated OSA was associated with a 5 -fold higher risk of incident CHD in the unadjusted model (HR: 5.11, 95\% CI 4.91 to 5.32) and a 3.5-fold higher risk in the fully adjusted model (HR: 3.54, 95\% CI 3.40 to 3.69) (figure 3A). Treated OSA was associated with a nearly fivefold higher risk for incident CHD in the unadjusted model (HR: $4.77,95 \%$ CI 4.10 to 5.55 ) and threefold higher risk in the fully adjusted model (HR: $3.06,95 \%$ CI 2.62 to 3.56 ) (figure 3B). Similar results were present in all subgroups (see online supplementary figure S2) and in our competing risk regression model (see online supplementary figure S1 and table S5).

\section{Incident stroke}

There were 50333 incident strokes $(1.8 \%$, event rate 3.07 (3.04-3.10)/1000 patient-years) in the OSA-negative group, 122 incident strokes $(9.2 \%, 14.6(12.2-17.4) / 1000$ patient-years) in the treated OSA group and 1601 incident strokes $(8.1 \%, 13.3$ $(12.7-14.0) / 1000$ patient-years) in the untreated OSA group. Figures 2 and 3 show the association between diagnoses of incident OSA and incident stroke in unadjusted and adjusted models. Untreated OSA was associated with 4-fold higher risk of incident stroke in the unadjusted (HR: 4.07, 95\% CI 3.87 to 4.28) and 3.5-fold higher risk in the fully adjusted model (HR:

Table 2 Predictors of a diagnosis of incident obstructive sleep apnoea using logistic regression analysis

\begin{tabular}{|c|c|c|}
\hline & OR & $95 \% \mathrm{Cl}$ of $\mathrm{OR}$ \\
\hline Age (+10-year) & 0.90 & 0.89 to 0.91 \\
\hline Gender: female vs male (ref.) & 0.49 & 0.45 to 0.53 \\
\hline \multicolumn{3}{|l|}{ Race } \\
\hline White (ref.) & 1.00 & 1.00 to 1.00 \\
\hline African-American & 0.90 & 0.86 to 0.93 \\
\hline Hispanic & 0.86 & 0.77 to 0.95 \\
\hline Other Race & 0.95 & 0.86 to 1.05 \\
\hline Income (+1 log) & 0.94 & 0.93 to 0.96 \\
\hline Marital status: unmarried vs married (ref.) & 1.12 & 1.09 to 1.16 \\
\hline Baseline eGFR (+10 mL/min. $\left./ 1.73 \mathrm{~m}^{2}\right)$ & 1.06 & 1.05 to 1.07 \\
\hline Presence of diabetes vs absence of diabetes (ref.) & 1.36 & 1.32 to 1.40 \\
\hline Presence of hypertension vs absence of hypertension (ref.) & 1.33 & 1.28 to 1.37 \\
\hline Presence of cardiovascular disease ${ }^{*}$ vs absence of cardiovascular disease* (ref.) & 1.35 & 1.30 to 1.40 \\
\hline Presence of congestive heart failure vs absence of congestive heart failure (ref.) & 1.97 & 1.88 to 2.06 \\
\hline Presence of cerebrovascular disease vs absence of cerebrovascular disease (ref.) & 1.26 & 1.20 to 1.33 \\
\hline Presence of peripheral arterial disease vs absence of peripheral arterial disease (ref.) & 1.29 & 1.23 to 1.36 \\
\hline Presence of chronic lung disease vs absence of chronic lung disease (ref.) & 1.91 & 1.85 to 1.98 \\
\hline Presence of dementia vs absence of dementia (ref.) & 1.06 & 0.92 to 1.23 \\
\hline Presence of rheumatological disease vs absence of rheumatological disease (ref.) & 1.36 & 1.22 to 1.51 \\
\hline Presence of malignancy vs absence of malignancy (ref.) & 1.05 & 1.00 to 1.10 \\
\hline Presence of AIDS/HIV vs absence of AIDS/HIV (ref.) & 0.93 & 0.76 to 1.14 \\
\hline Presence of depression vs absence of depression (ref.) & 1.71 & 1.64 to 1.78 \\
\hline Body mass index $\left(+1 \mathrm{~kg} / \mathrm{m}^{2}\right)$ & 1.14 & 1.13 to 1.14 \\
\hline
\end{tabular}

${ }^{*}$ Cardiovascular disease was defined as acute myocardial infraction, angina, coronary artery disease, previous coronary artery bypass grafting or percutaneous coronary intervention. eGFR, estimated glomerular filtration rate. 

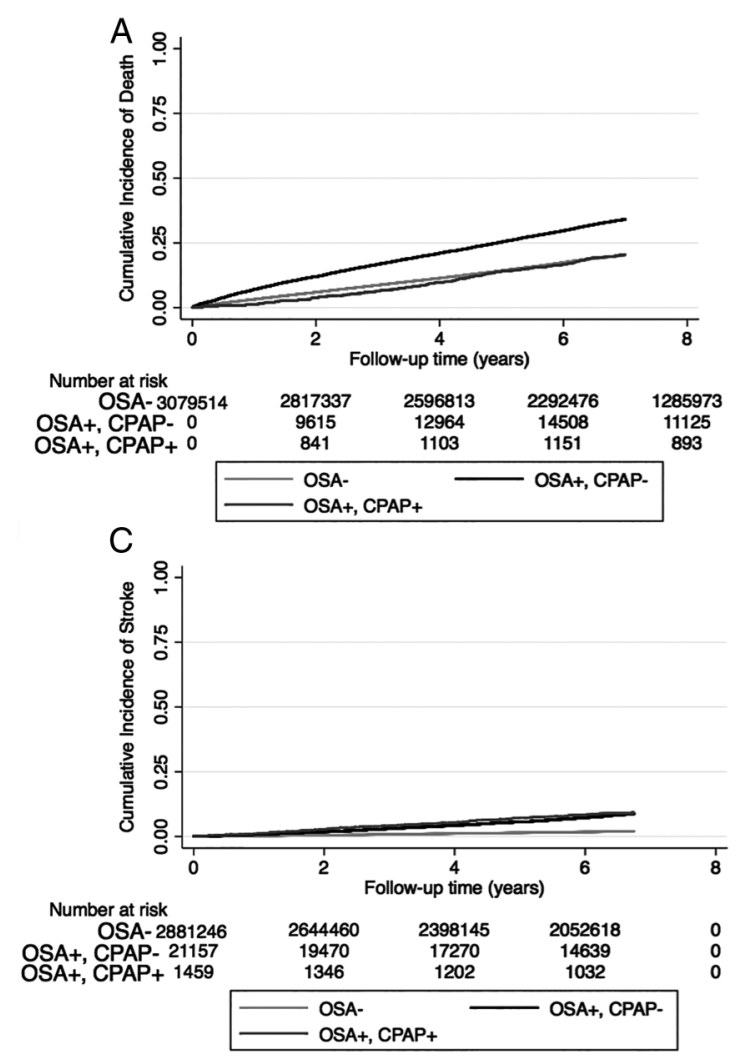
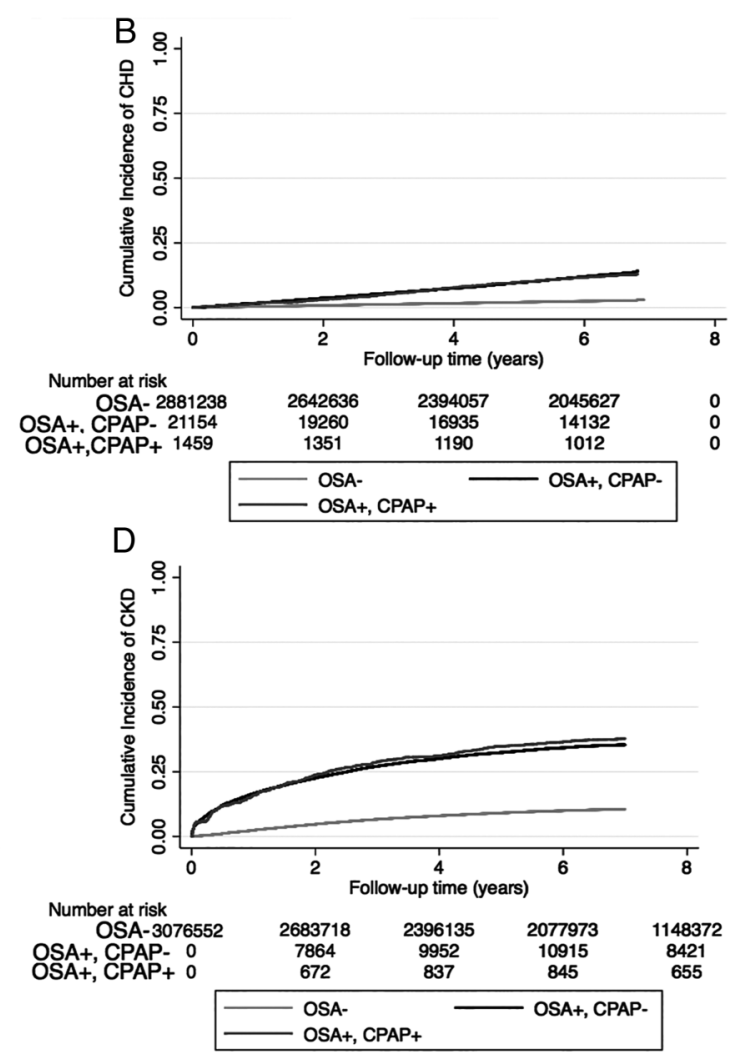

Figure 2 Association between a diagnosis of incident obstructive sleep apnoea (OSA) and outcomes ((A) cumulative incidence of death; (B) cumulative incidence of coronary heart disease, CHD; (C) cumulative incidence of ischaemic stroke event and (D) cumulative incidence of chronic kidney disease (CKD), using Kaplan-Meier curves.

3.48 , 95\% CI 3.28 to 3.64 ) (figure $3 \mathrm{~A}$ ). Treated OSA was associated with a nearly 4-fold higher risk for incident stroke in the unadjusted model (HR: 4.38, 95\% CI 3.67 to 5.23) and 3.5 -fold higher risk in the fully adjusted model (adjusted HR: 3.50 , 95\% CI 2.92 to 4.19 ) (figure 3B). Similar results were found in all subgroups (see online supplementary figure S3) and in our competing risk regression model (see online supplementary figure S1 and table S5).

\section{Incidence of eGFR $<60 \mathrm{~mL} / \mathrm{min} / 1.73 \mathrm{~m}^{2}$}

There were 290037 incident CKD events (10\%, event rate 15.05 (14.99-15.11)/1000 patient-years) in the OSA-negative group, 434 incident CKD events (29\%, 46.26 (41.19-51.96)/ 1000 patient-years) in the treated OSA group and 5486 incident CKD events (25\%, 38.31 (36.93-39.74)/1000 patient-years) in the untreated OSA group. Figures 2 and 3 show the associations between a diagnosis of incident OSA and incident CKD in unadjusted and adjusted models. Untreated OSA was associated with a threefold higher risk of incident CKD in the unadjusted model (HR: 3.25, 95\% CI 3.13 to 3.37) and a twofold higher risk in the fully adjusted model (HR: $2.27,95 \%$ CI 2.19 to 2.36 ) (figure 3A). Treated OSA was associated with a nearly 3.8 -fold higher risk of incident CKD in the unadjusted model (HR: 3.76, 95\% CI 3.35 to 4.23 ) and a 2.8 -fold higher risk in the fully adjusted model (HR: 2.79 , 95\% CI 2.48 to 3.13 ) (figure 3B). Similar results were found in all subgroups (see online supplementary figure S4) and in our competing risk regression model (see online supplementary figure S1 and table S5).

\section{Deterioration of kidney function}

The median (IQR) of the eGFR slope was $-0.41(-2.01$ to $0.99) ;-0.61(-2.69$ to 0.93$)$ and $-0.87(-3.00$ to 0.70$)$ in
OSA-negative, untreated OSA-positive and treated OSA-positive patients, respectively. Of the veterans, 292825 had slopes steeper than $-5 \mathrm{~mL} / \mathrm{min} / 1.73 \mathrm{~m}^{2} /$ year. Figure 3 shows the association between a diagnosis of incident OSA status and the slope of deterioration of the kidney function. Untreated OSA-positive patients had a significantly higher risk of rapid deterioration of kidney function in the unadjusted model (OR: 1.34, 95\% CI 1.29 to 1.39 ) and the fully adjusted model (OR: $1.30,95 \% \mathrm{CI}$ 1.24 to 1.35 ) (figure $3 \mathrm{~A}$ ). Treated OSA-positive patients also had a significantly higher risk of rapid deterioration of kidney function in the unadjusted model (OR: $1.28,95 \%$ CI 1.10 to 1.49 ) and the fully adjusted model (OR: $1.28,95 \%$ CI 1.09 to 1.50 ) (figure $3 \mathrm{~B}$ ). Similar results were found in most subgroups (see online supplementary figure S5).

In sensitivity analyses using polysomnography as secondary criteria of the diagnosis instead of the ICD-9-CM diagnosis code, qualitatively and quantitatively similar results were found (results not shown).

\section{DISCUSSION}

In a large cohort of US veterans with baseline eGFR $\geq 60 \mathrm{~mL} /$ $\min / 1.73 \mathrm{~m}^{2}$, we examined the association of a diagnosis of incident untreated/treated OSA with higher risk of all-cause and post-ischaemic event mortality, incident $\mathrm{CHD}$ and stroke, incident eGFR $<60 \mathrm{~mL} / \mathrm{min} / 1.73 \mathrm{~m}^{2}$ and the rate of kidney function decline. Incident diagnosis of OSA was associated with a higher risk of all clinical outcomes.

Higher mortality associated with OSA was reported in several previous studies, which used the gold standard of polysomnography for diagnosing OSA, ${ }^{2-4}{ }^{11}$ but some studies performed in prevalent elderly patients did not confirm this association. ${ }^{5-7}$ This discrepancy could be explained by survival bias, ${ }^{5-7}$ or it 
Panel A - CPAP negative patients

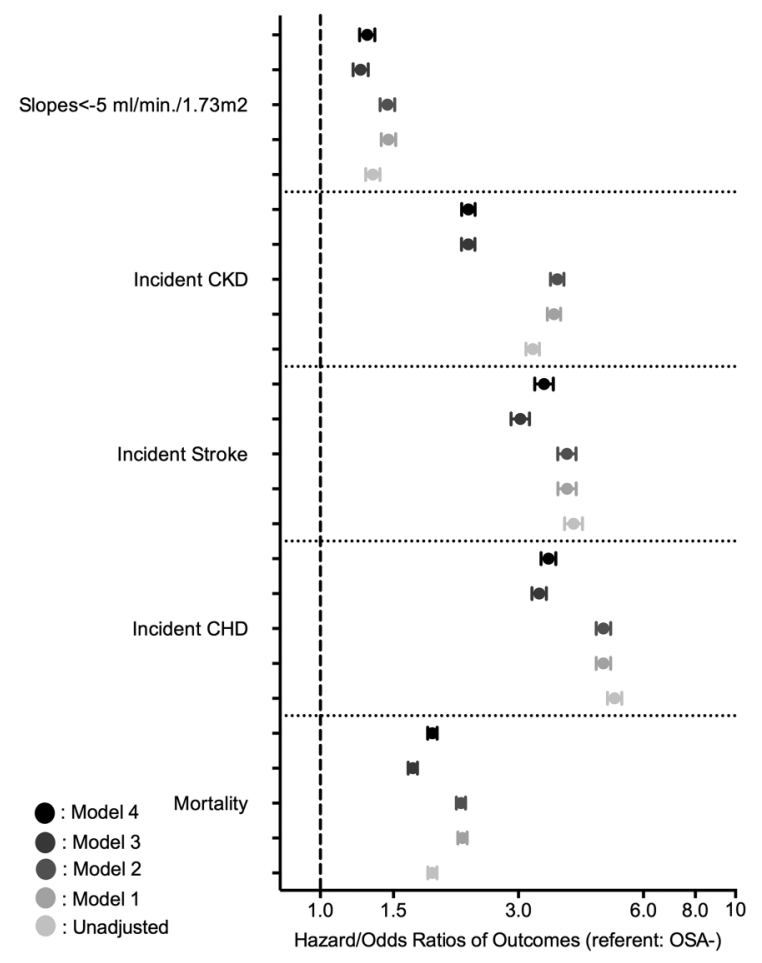

Panel B - CPAP positive patients

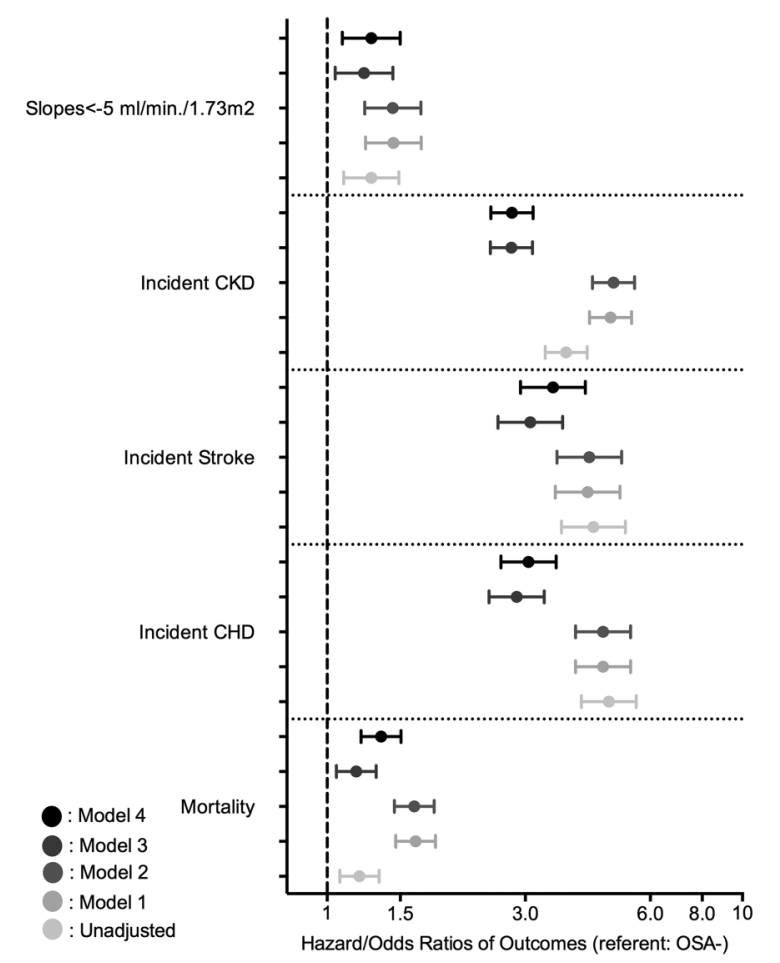

Figure 3 Association between a diagnosis of incident obstructive sleep apnoea (OSA) ((A) without CPAP treatment; (B) with CPAP treatment) and outcomes using time-dependent Cox regression (mortality and incident chronic kidney disease, CKD), Cox proportional regression (incident coronary heart disease, CHD and ischaemic stroke event) and logistic regression analysis (for slope) compared with OSA-negative patients model 1: adjusted for age, gender, race/ethnicity; model 2: adjusted for model 1 variables and baseline estimated glomerular filtration rate (eGFR); model 3: adjusted for model 2 variables and comorbidities at baseline (diabetes, hypertension, cardiovascular disease, congestive heart failure, cerebrovascular disease, peripheral vascular disease, lung disease, dementia, rheumatic disease, malignancy, HIVIAIDS and depression) and measures of quality of care (number of administered cholesterol measurements and influenza vaccinations); model 4: adjusted for model 3 variables and income, marital status and body mass index.

could be related to the pathophysiology of OSA. There is increasing evidence showing that patients with OSA can have a survival advantage secondary to ischaemic preconditioning. ${ }^{26-30}$ The defense mechanism for the repetitive milder ischaemic events during OSA can result in structural and functional changes in brain cells and myocardial cells, preparing them for survival in the face of a major ischaemic event through a mechanism of ischaemic preconditioning, ${ }^{28-30}$ however, this seems not to protect the kidney. ${ }^{31}$ Our postischaemic event mortality analyses support this hypothesis. When comparing the effect sizes of all-cause mortality versus postevent mortality, those for untreated patients with OSA were significantly smaller and became non-significant in treated patients with OSA. This observation supports the hypothesis that 'well-prepared' patients may have a better chance to survive a major ischaemic event, while those without an ischaemic preconditioning defense mechanism may have higher postevent mortality. This can result in a selection bias in studies examining the elderly, which would have an accumulation of these survivors in their study population. This hypothesis needs to be tested in the future.

The associations of incident diagnosis of OSA with higher risk of incident CHD and stroke can be explained by increased sympathetic activity, intermittent hypoxia, hypertension, accelerated atherosclerosis or a combination of these in patients suffering from OSA. Our observations support those made in previous smaller studies. ${ }^{3} 3233$

We report strong associations of incident diagnosis of OSA with incident low eGFR and progressive loss of kidney function. OSA can cause increased renal sympathetic activity, intermittent hypoxia, hypertension, accelerated atherosclerosis, production of proinflammatory cytokines, endothelial dysfunction and proteinuria, which could all contribute to the development and progression of CKD. Previous studies have reported a significant association of OSA with the progression of kidney disease. ${ }^{34} 35$ In a study comparing 27 patients with OSA with 32 healthy controls, those with OSA had higher filtration fraction suggesting the presence of glomerular hyperfiltration. ${ }^{13}$ Short-term CPAP therapy significantly decreased the filtration fraction, suggesting that CPAP may prevent nephropathy by decreasing OSA-related glomerular hyperfiltration. ${ }^{13}$ A recent study from Taiwan reported a two times higher CKD and ESRD risk in patients with OSA compared with OSA-free counterparts. ${ }^{16}$

Our study is notable for its large sample size and event numbers, and for it being representative of veterans who received care in the VA system in the entire USA. To our knowledge, this is the largest study to find substantial associations between a diagnosis of incident OSA and kidney function decline and incident decrease in eGFR. This study also has several limitations that need to be acknowledged. This being an observational study, we can only report associations, and we cannot claim that OSA was indeed the cause of the worse clinical outcomes. Additionally, models could only be adjusted for identified confounders for which we had available data. Therefore, we cannot rule out residual confounding. Our study is limited by the use of diagnostic codes to define OSA, CPAP treatment and polysomnography. The diagnostic performance of these codes is not known. However, the significant predictors of incident diagnosis of OSA in our study were similar to those 
Figure 4 Association between a diagnosis of incident obstructive sleep apnoea (OSA) and post- coronary heart disease (CHD)/post-ischaemic stroke event mortality using Cox proportional regression analysis compared with OSA-negative patients. model 1: adjusted for age, gender, race/ ethnicity; model 2: adjusted for model 1 variables and baseline estimated glomerular filtration rate (eGFR); model hypertension, cardiovascular disease, congestive heart failure, cerebrovascular disease, peripheral vascular disease, lung disease, dementia, rheumatic disease, malignancy, HIVIAIDS and depression) and measures of quality of care (number of administered cholesterol measurements and influenza vaccinations); model 4: adjusted for model 3 variables and income, marital status and body mass index. 3: adjusted for model 2 variables and comorbidities at baseline (diabetes,

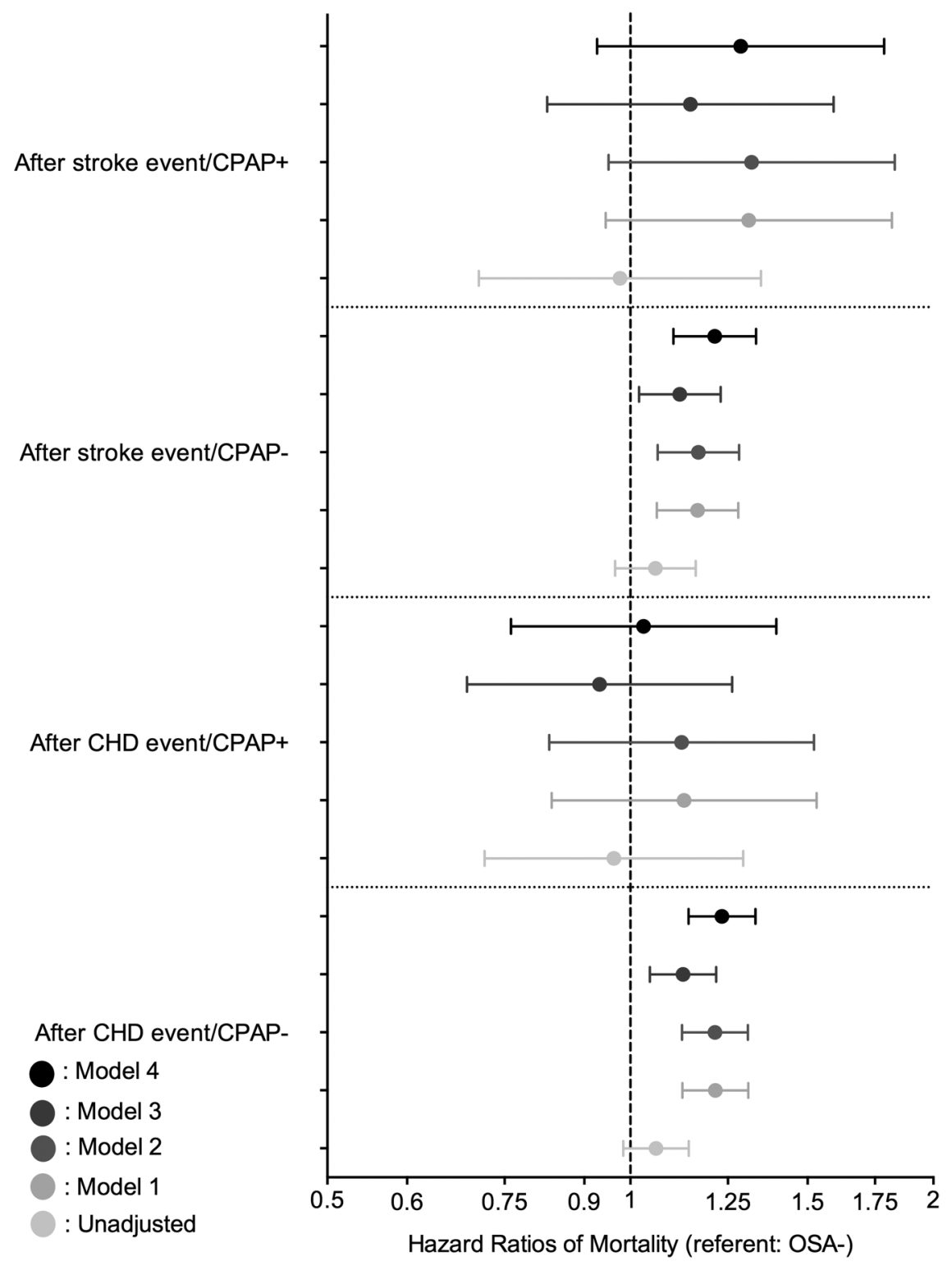

found in previous studies. ${ }^{36}$ Moreover, sensitivity analyses using polysomnography to define OSA led to similar results. We were unable to assess the associations of OSA severity with various outcomes as we did not have detailed polysomnography data. The proportion of patients who received CPAP treatment in our study was smaller than expected, which raises the possibility that patients received CPAP treatment outside the VA health system, refused CPAP treatment, or had mild/positional OSA, or received surgical treatment, or received CPAP treatment after the end of our follow-up period. Moreover, our study population consisted of mostly male US veterans, so the result should be applied with caution to female patients and to patients in other countries. Because we did not have information about causes of death, we could not analyse associations with causespecific mortality. Additionally, we did not have data about albuminuria in our database; consequently we can only comment on associations between OSA and kidney function decline, but not on associations with properly defined incident CKD.

\section{CONCLUSIONS}

In our large and contemporary cohort of more than three million US veterans, a diagnosis of incident OSA was associated with higher risk of mortality, incident $\mathrm{CHD}$, stroke and $\mathrm{CKD}$, and faster kidney function decline. Improvement of the diagnostics and early detection as well the effect of proper therapy for OSA on preventing these clinical events need to be tested in clinical trials.

\section{Author affiliations}

${ }^{1}$ Division of Nephrology, Department of Medicine, University of Tennessee Health Science Center, Memphis, Tennessee, USA

${ }^{2}$ Department of Medicine, Division of Nephrology, University Health Network, University of Toronto, Toronto, Canada

${ }^{3}$ Institute of Behavioral Sciences, Semmelweis University, Budapest, Hungary

${ }^{4}$ Department of Psychiatry, University Health Network, University of Toronto,

${ }^{5}$ Department of Cardiothoracic Surgery and Cardiothoracic Anesthesia, Linköping University Hospital, Linköping, Sweden

${ }^{6}$ Division of Cardiovascular Medicine, Department of Medical and Health Sciences, Linköping University, Linköping, Sweden

${ }^{7}$ Pulmonary Section, Memphis Veterans Affairs Medical Center, Memphis, Tennessee, USA

${ }^{8}$ Nephrology Section, Memphis Veterans Affairs Medical Center, Memphis, Tennessee, USA

${ }^{9}$ Department of Epidemiology, Fielding School of Public Health, University of California, Los Angeles (UCLA), Los Angeles, California, USA

${ }^{10}$ Division of Nephrology, University of Virginia, Charlottesville, Virginia, USA Toronto, Canada 
${ }^{11}$ Kaiser Permanente, Los Angeles, California, USA

${ }^{12}$ Division of Nephrology, University of California, Irvine, California, USA

Contributors Conception and design: MZM, IM and CPK. Analysis and interpretation: MZM, OAA, JZM and CPK. Drafting the manuscript for important intellectual content: MZM, IM, MN, ZS, AXF, KMH, JLL, JJS, ES, KK-Z and CPK. Writing the manuscript: MZM and CPK.

Funding This study is supported by grant 1R01DK096920 from the NIH to CPK and KK-Z, and by resources from the US Department of Veterans Affairs. Support for VA/CMS data is provided by the Department of Veterans Affairs, Veterans Health Administration, Office of Research and Development, Health Services Research and Development, VA Information Resource Center (Project Numbers SDR 02-237 and 98-004). OAA was supported by Veni career grant 916.96 .059 from the Netherlands Organisation for Scientific Research.

Competing interests $\mathrm{CPK}, \mathrm{AXF}, \mathrm{KK}-\mathrm{Z}$ and $\mathrm{KMH}$ are employees of the Department of Veterans affairs. Opinions expressed in this paper are those of the authors' and do not necessarily represent the opinion of the Department of Veterans Affairs. The results of this paper have not been published previously in whole or part.

Ethics approval The institutional review committees at the Memphis and Long Beach Veterans Affairs Medical Centers.

Provenance and peer review Not commissioned; externally peer reviewed.

\section{REFERENCES}

1 Peppard PE, Young T, Barnet JH, et al. Increased prevalence of sleep-disordered breathing in adults. Am J Epidemiol 2013;177:1006-14.

2 Young T, Finn L, Peppard PE, et al. Sleep disordered breathing and mortality: eighteen-year follow-up of the wisconsin sleep cohort. Sleep 2008;31:1071-8.

3 Yaggi HK, Concato J, Kernan WN, et al. Obstructive sleep apnea as a risk factor for stroke and death. N Engl J Med 2005;353:2034-41.

4 Marshall NS, Wong KK, Cullen SR, et al. Sleep apnea and 20-year follow-up for all-cause mortality, stroke, and cancer incidence and mortality in the Busselton Health Study cohort. I Clin Sleep Med 2014;10:355-62.

5 Lavie P, Lavie L. Unexpected survival advantage in elderly people with moderate sleep apnoea. J Sleep Res 2009;18:397-403.

6 Rich J, Raviv A, Raviv N, et al. All-cause mortality and obstructive sleep apnea severity revisited. Otolaryngol Head Neck Surg 2012;147:583-7.

7 Johansson P, Alehagen U, Svanborg E, et al. Clinical characteristics and mortality risk in relation to obstructive and central sleep apnoea in community-dwelling elderly individuals: a 7-year follow-up. Age Ageing 2012;41:468-74.

8 Seicean S, Strohl KP, Seicean A, et al. Sleep disordered breathing as a risk of cardiac events in subjects with diabetes mellitus and normal exercise echocardiographic findings. Am I Cardiol 2013;111:1214-20.

9 Punjabi NM, Caffo BS, Goodwin JL, et al. Sleep-disordered breathing and mortality: a prospective cohort study. PLoS Med 2009;6:e1000132.

10 Redline S, Yenokyan G, Gottlieb DJ, et al. Obstructive sleep apnea-hypopnea and incident stroke: the sleep heart health study. Am J Respir Crit Care Med 2010;182:269-77.

11 Ge X, Han F, Huang Y, et al. Is obstructive sleep apnea associated with cardiovascular and all-cause mortality? PlOS ONE 2013;8:e69432.

12 de Oliveira Rodrigues CJ, Marson O, Tufic S, et al. Relationship among end-stage renal disease, hypertension, and sleep apnea in nondiabetic dialysis patients. Am J Hypertens 2005;18(2 Pt 1):152-7.

13 Kinebuchi S, Kazama JJ, Satoh M, et al. Short-term use of continuous positive airway pressure ameliorates glomerular hyperfiltration in patients with obstructive sleep apnoea syndrome. Clin Sci (Lond) 2004;107:317-22.

14 Molnar MZ, Lazar AS, Lindner A, et al. Sleep apnea is associated with cardiovascular risk factors among kidney transplant patients. Clin J Am Soc Nephrol 2010;5:125-32.
15 Fornadi K, Ronai KZ, Turanyi CZ, et al. Sleep apnea is not associated with worse outcomes in kidney transplant recipients. Sci Rep 2014;4:6987.

16 Lee YC, Hung SY, Wang HK, et al. Sleep apnea and the risk of chronic kidney disease: a nationwide population-based cohort study. Sleep 2015;38:213-21

17 Molnar MZ, Alhourani HM, Wall BM, et al. Association of hepatitis C viral infection with incidence and progression of chronic kidney disease in a large cohort of US veterans. Hepatology 2015;61:1495-502.

18 Gosmanova EO, Lu JL, Streja E, et al. Association of medical treatment nonadherence with all-cause mortality in newly treated hypertensive US veterans. Hypertension 2014;64:951-7.

19 VIReC Research User Guide. VHA Medical SAS Inpatient Datasets FY 2006-2007. Hines IUSDoVAVIRC. 2007

20 Molnar MZ, Kalantar-Zadeh K, Lott EH, et al. ACE inhibitor and angiotensin receptor blocker use and mortality in patients with chronic kidney disease. J Am Coll Cardiol 2014;63:650-8.

21 Kovesdy CP, Lott EH, Lu JL, et al. Hyponatremia, hypernatremia, and mortality in patients with chronic kidney disease with and without congestive heart failure. Circulation 2012:125:677-84.

22 Kovesdy CP, Lott EH, Lu JL, et al. Outcomes associated with microalbuminuria: effect modification by chronic kidney disease. J Am Coll Cardiol 2013;61: 1626-33.

23 VIReC Research User Guide. VHA Medical SAS Inpatient Datasets FY2006-2007. Hines, IL: US Department of Veterans Affairs VA Information Resource Center, 2007.

24 US Department of Veterans Affairs VA Information Resource Center Data Quality Update: Race, 2009.

25 Fine J, Gray R. A proportional hazards model for subdistribution of a competing risk. J Am Stat Assoc 1999;94:496-509.

26 Bartlett DJ, Rae C, Thompson $\mathrm{CH}$, et al. Hippocampal area metabolites relate to severity and cognitive function in obstructive sleep apnea. Sleep Med 2004;5:593-6.

27 Lavie L. Oxidative stress in obstructive sleep apnea and intermittent hypoxiarevisited - the bad ugly and good: implications to the heart and brain. Sleep Med Rev 2015;20:27-45.

28 Rosenzweig I, Kempton MJ, Crum WR, et al. Hippocampal hypertrophy and sleep apnea: a role for the ischemic preconditioning? PlOS ONE 2013; 8:e83173.

29 Rosenzweig I, Williams SC, Morrell MJ. The impact of sleep and hypoxia on the brain: potential mechanisms for the effects of obstructive sleep apnea. Curr Opin Pulm Med 2014;20:565-71.

30 Shah N, Redline S, Yaggi HK, et al. Obstructive sleep apnea and acute myocardial infarction severity: ischemic preconditioning? Sleep Breath 2013;17: 819-26.

31 Eltzschig HK, Eckle T. Ischemia and reperfusion-from mechanism to translation. Nat Med 2011:17:1391-401.

32 Joo BE, Seok HY, Yu SW, et al. Prevalence of sleep-disordered breathing in acute ischemic stroke as determined using a portable sleep apnea monitoring device in Korean subjects. Sleep Breat 2011;15:77-82.

33 Arzt $\mathrm{M}$, Young $\mathrm{T}$, Finn $\mathrm{L}$, et al. Association of sleep-disordered breathing and the occurrence of stroke. Am J Respir Crit Care Med 2005;172:1447-51.

34 Szentkiralyi A, Czira ME, Molnar MZ, et al. High risk of obstructive sleep apnea is a risk factor of death censored graft loss in kidney transplant recipients: an observational cohort study. Sleep Med 2011;12:267-73.

35 Stehouwer $C D$, Gall MA, Twisk JW, et al. Increased urinary albumin excretion, endothelial dysfunction, and chronic low-grade inflammation in type 2 diabetes: progressive, interrelated, and independently associated with risk of death. Diabetes 2002;51:1157-65.

36 Sharma SK, Malik V, Vasudev C, et al. Prediction of obstructive sleep apnea in patients presenting to a tertiary care center. Sleep Breat 2006;10:147-54. 